\title{
Dejar de fumar en una persona con enfermedad física grave: a propósito de un caso
}

\author{
Carmela Martínez-Vispo, Ana López-Durán, Elisardo Becoña \\ Unidad de Tabaquismo y Trastornos Adictivos, Departamento de Psicología Clínica \\ y Psicobiología, Facultad de Psicología, Universidad de Santiago de Compostela
}

\section{RESUMEN}

Fumar es uno de los factores de riesgo más importantes para la salud y calidad de vida de las personas, especialmente cuando está presente una enfermedad respiratoria y/o cardiovascular. Se presenta la evaluación e intervención psicológica para dejar de fumar de un varón de 41 años de edad con enfermedad física grave y con un consumo diario de 20 cigarrillos y 5 puritos. Se aplicó un programa cognitivo-conductual para dejar de fumar de seis sesiones individuales. Al final de la intervención psicológica la persona dejó de fumar y se mantuvo abstinente en los seguimientos realizados tras finalizar el tratamiento (a los 3, 6 y 12 meses), mostrando una mejora en los problemas físicos que presentaba. Se concluye que se puede dejar de fumar con una intervención psicológica adecuada logrando con ello una clara mejora en la salud, especialmente cuando se trata de personas con problemas físicos graves.

Palabras Clave

Intervención psicológica; dejar de fumar; enfermedad física.

\section{ABSTRACT}

Smoking is an important risk factor for health and quality of life, especially when there is a respiratory and/or cardiovascular disease. We present the evaluation and psychological intervention to quit smoking of a 41 years old male with severe physical illness and a daily consumption of 20 cigarettes and 5 small cigars. We applied a cognitive-behavioural smoking cessation program with six individual sessions. At the end of the psychological intervention the person quitted smoking and remained abstinent at 3, 6 and 12 months follow-ups, showing an improvement in the physical problems presented. We conclude that anyone can quit smoking with an appropriate psychological intervention thereby achieving a significant improvement in health, especially when there are severe physical problems.

KEYWORDS

Psychological intervention; smoking cessation; physical illness.

Recibido: 07/01/2019; aceptado: 13/05/2019.

Los autores de este artículo declaran que no tienen ningún conflicto de interés.

Correspondencia: Unidad de Tabaquismo y Trastornos Adictivos, Universidad de Santiago de Compostela, Facultad de Psicología, Departamento de Psicología Clínica y Psicobiología, Campus Vida, 15782 Santiago de Compostela, España. 
Fumar constituye uno de los mayores problemas sanitarios a los que nos enfrentamos en la actualidad, ya que es la principal causa evitable de morbilidad y mortalidad prematura en el mundo (USDHHS, 2014). En España, aunque se ha reducido de manera importante la prevalencia del consumo de tabaco, todavía es uno de los países europeos con mayor porcentaje de población fumadora (OECD, 2013), ya que un $22,1 \%$ de la población de 15 y más años fuma a diario según la última Encuesta Nacional de Salud (INE, 2018).

El tabaco es uno de los principales factores de riesgo que inciden en el inicio y pronóstico de las enfermedades respiratorias y cardiovasculares. De hecho, se le considera responsable de al menos el 36\% de las muertes debidas a enfermedades respiratorias no contagiosas (Jha et al., 2013) y del 30\% de las muertes por enfermedades cardiovasculares como la enfermedad cerebrovascular, la arteriosclerosis, la enfermedad cardíaca isquémica y el síndrome coronario agudo (Messner \& Bernhard, 2014). El riesgo de sufrir una enfermedad coronaria se incrementa notablemente incluso con exposiciones bajas al tabaco. Por ejemplo, en el estudio llevado a cabo por Yusuf et al. (2004), en el que se pretendía estudiar los factores de riesgo modificables para las enfermedades cardiovasculares en más de 15.000 casos y controles de 52 países, encontraron que fumar incluso tan solo cinco cigarrillos al día aumentaba el riesgo de sufrir problemas cardiovasculares, lo que indica que no existe un nivel seguro de fumar.

Además, se sabe que existe una relación entre el consumo de tabaco, los síntomas depresivos y las enfermedades coronarias (Penninx,
2017; Watkins, et al., 2013). Por ejemplo, Doyle et al. (2014), realizaron una revisión sistemática acerca de la asociación entre depresión y dejar de fumar en personas con enfermedad coronaria, y encontraron que la sintomatología depresiva estaba asociada con una menor probabilidad de dejar de fumar en este grupo de pacientes. Dada la relevancia de dichos síntomas en la evolución y pronóstico de dichas enfermedades, los resultados de estos estudios sugieren que la evaluación y seguimiento de los síntomas depresivos en estos pacientes debe realizarse de manera rutinaria, y deben ser tenidos en cuenta a lo largo de su proceso de recuperación.

Por otro lado, en la actualidad existen intervenciones psicológicas eficaces para lograr la abstinencia del tabaco. De hecho, la evidencia empírica muestra que el abordaje psicológico de la dependencia del tabaco mejora de manera sustancial el logro de la abstinencia y su mantenimiento a largo plazo, considerándose como intervenciones de primera elección (Hartmann-Boyce, Stead, Cahill, \& Lancaster, 2013; Patnode et al., 2015; Siu, 2015).

A pesar de estos datos, también se ha encontrado en los últimos años una reducción de los porcentajes de abstinencia tras haber recibido intervención psicológica y/o farmacológica para dejar de fumar (Becoña, LópezDuran, Fernandez del Rio, \& Martínez, 2014; Irvin, Hendricks, \& Brandon 2003; Irvin \& Brandon, 2000). Diferentes estudios sugieren que este fenómeno podría deberse a que las personas que continúan fumando y demandan intervención para dejar de fumar tienen una mayor dependencia de la nicotina y mayor 
psicopatología en general, lo que implica una mayor dificultad a la hora de abordar el logro y mantenimiento de la abstinencia (Becoña, et al. 2014; Leyro et al. 2016).

Cuando las enfermedades respiratorias y cardiovasculares están presentes y además, coocurren, dejar de fumar resulta la medida más eficaz para mejorar el pronóstico, prevenir complicaciones físicas y mejorar la salud y calidad de vida de las personas que las padecen. En el presente estudio de caso se presenta el caso de un varón que sufre ambas condiciones y que solicita una intervención psicológica para dejar de fumar.

\section{Método}

\section{Identificación y motivo de consulta}

Se presenta el caso de un varón de 41 años de edad con enfermedad física grave (angina de pecho y bronconeumonía) y con un consumo diario de 20 cigarrillos de 0,9 mg de nicotina y 5 puritos, que solicita intervención para dejar de fumar en la Unidad de Tabaquismo y Trastornos Adictivos de la Universidad de Santiago de Compostela.

\section{Historia del problema}

F. M. probó su primer cigarrillo a los 17 años y empezó a fumar de forma regular a los 18 años, por lo que lleva 23 años fumando. Inhala el humo al fumar, da bastantes caladas a cada cigarrillo y los fuma enteros hasta el filtro. En casa convive con su pareja y su sobrino, el cual también fuma. Considera que fumar cigarrillos afecta en gran medida a la salud y su médico le ha recomendado en repetidas ocasiones que debe dejar de fumar dado que padece proble- mas cardiovasculares y, además, en el último año ha sufrido varios episodios de bronconeumonía. Refiere también que el tabaco le produce otras molestias físicas importantes como secreciones mucosas, tos, cansancio y una reducción notable del rendimiento cuando realiza actividad física.

A lo largo de su vida sólo ha realizado un intento serio de dejar de fumar que tuvo lugar 4 meses antes de acudir a la Unidad y tras haber sufrido un episodio coronario agudo (angina de pecho). En dicho intento logró mantenerse sin fumar durante 3 días, experimentando intensos síntomas del síndrome de abstinencia como irritabilidad, ansiedad, inquietud, aumento del apetito y frustración, que le llevaron a fumar de nuevo para conseguir aliviar el malestar que le provocaban. Además, en las semanas previas a demandar intervención, había sufrido un episodio de bronconeumonía por el que había sido hospitalizado, lo que le lleva a tomar la decisión de dejar de fumar y solicitar ayuda para ello.

\section{Procedimiento}

Previo al inicio de la intervención, se le explicó el protocolo de evaluación, intervención, y seguimientos y se firmó el consentimiento informado. Se llevaron a cabo dos sesiones de evaluación pretratamiento, con el objetivo de que la duración de dichas sesiones no fuese superior a una hora, y también que F. M. dispusiese de una semana para realizar los autorregistros del consumo de tabaco entre una y otra sesión y así poder establecer la línea base del consumo.

Con respecto a la intervención, consta de 6 sesiones individuales de una hora de duración. 
Una vez finalizada la intervención, se realizaron seguimientos presenciales a los 3, 6 y 12 meses (Velicer, Prochaska, Rossi, \& Snow, 1992). En cada uno de los seguimientos se administró un cuestionario para evaluar las situaciones de riesgo de recaída, las estrategias usadas en dichas situaciones, los beneficios obtenidos al dejar de fumar y la motivación para mantenerse abstinente, entre otras variables (Becoña \& Míguez, 1995). También se administró el BDI-II en la última sesión de tratamiento, así como en los seguimientos, dada la relevancia de la sintomatología depresiva en la evolución y pronóstico de las enfermedades cardiovasculares y su relación con el consumo de tabaco (Doyle et al., 2014).

Además, en la evaluación, en las sesiones de intervención y durante los seguimientos se evaluó el nivel de monóxido de carbono en aire espirado (CO). Esta medición permite dar feedback al individuo acerca de su consumo mientras todavía fuma, así como permite la validación bioquímica de la abstinencia autoinformada cuando deja de fumar. La abstinencia se considera confirmada con valores inferiores a 10 p.p.m. (West, Hajek, Stead, \& Stapleton, 2005).

También se realizó la medición del peso, la tensión arterial y la frecuencia cardíaca, tanto en la evaluación pretratamiento, como en la última sesión de intervención y en los seguimientos realizados a los 3, 6 y 12 meses.

\section{Evaluación}

\section{Instrumentos}

En la sesión de evaluación se realizó una entrevista clínica con el objetivo de evaluar variables relacionadas con el consumo de tabaco, intentos de abandono previos o reduc- ción de cigarrillos, las razones para dejar de fumar en otros intentos, razones para dejarlo en el momento de la evaluación, algún cambio físico o/y psicológico cuando la persona ha intentado dejar de fumar, si ha acudido a algún tratamiento previo para dejar de fumar, procedimientos que ha seguido para dejar de fumar, causas de la recaída en otros intentos, apoyo social, creencias sobre la incidencia del tabaco sobre la salud, síntomas y molestias que padecen o han padecido por fumar cigarrillos, consumo de alcohol, café, otras drogas y medicamentos, deseo de dejar de fumar y expectativas sobre el tratamiento.

La medición del nivel de monóxido de carbono en aire espirado (CO), se llevó a cabo con el instrumento Micro+ Smokerslyzer ${ }^{\circledast}$.

Con el objetivo de tener información más precisa respecto a variables relacionadas con el consumo de tabaco, así como variables psicológicas que podrían influir en el proceso de abandono de este caso concreto, se aplicaron los siguientes cuestionarios:

- Cuestionario sobre el Hábito de Fumar (Becoña, 1994), en el que se recoge información sobre variables sociodemográficas (sexo, edad, situación laboral, nivel de estudios) y características del consumo de tabaco (historia de consumo, número máximo de cigarrillos que llegó a fumar en un día, intentos previos de abandono, enfermedades y molestias generadas por el consumo).

- Escala para la Evaluación de los Estadios de Cambio (McConnaughy, Prochaska, $\&$ Velicer, 1983), con la que se evalúa en qué estadio de cambio se encuentra la 
persona: precontemplación (no intención de dejar de fumar en los próximos 6 meses), contemplación (intención de dejar de fumar en los próximos 6 meses), y preparación para la acción (intención de dejar de fumar en los próximos 30 días y han estado al menos un día abstinente en el año previo).

- Escala de Tentación a Fumar (forma corta; Velicer, DiClemente, Rossi, \& Prochaska 1990), con la que se trata de evaluar la tentación de fumar en (1) situaciones de afecto positivo/situación social; (2) situaciones de afecto negativo; y (3) situaciones de hábito/craving.

- Escala Breve del Síndrome de Dependencia de la Nicotina (NDSS-S; Becoña et al., 2011), que evalúa distintas facetas de la dependencia de la nicotina: el impulso, la prioridad, la tolerancia, la continuidad y la estereotipia.

- Cuestionario de Ansiedad Estado-Rasgo (STAI A-E y STAI A-R; Spielberger, Gorsuch \& Lushene, 1982). Instrumento que evalúa dos componentes de la ansiedad: (1) Ansiedad Rasgo, que es un factor de personalidad que comprende las diferencias individuales, relativamente estables, para responder ante situaciones percibidas como amenazantes; y (2) Ansiedad Estado, que sería una reacción de ansiedad referida a un período de tiempo transitorio y concreto.

- Inventario de Depresión de Beck, segunda versión (BDI-II; Sanz \& Vázquez, 2011). Este inventario persigue detectar la presencia y el grado de los síntomas depresivos.

\section{Resultados de la Evaluación}

En cuanto a los resultados obtenidos mediante la entrevista y la aplicación de los cuestionarios, su deseo de dejar de fumar y de realizar el programa para dejar de fumar era de 10 (en una escala de 0 a 10). En la evaluación de los estadios de cambio se sitúa en el de preparación para la acción ya que tiene la intención de dejarlo en los próximos 30 días y ha conseguido estar sin fumar más de un día en el último año. En la NDSS-S obtiene una puntuación de 17 , lo que indica dependencia de la nicotina (puntuación mayor de 11) (Becoña et al., 2011).

En la escala de tentación a fumar obtiene puntuaciones elevadas en sus tres subescalas, lo que muestra una gran tentación a fumar tanto en situaciones relacionadas con el afecto negativo, como en las que implican afecto positivo y craving/hábito.

En el STAI Rasgo obtiene una puntuación directa de 22 que lo sitúa en el centil 60 (por encima de la media de la población general), lo que refleja que es una persona que tiende a reaccionar de manera ansiosa ante muchas situaciones. En el STAI Estado su puntuación directa fue de 12 , centil 20 , lo que indica que en el momento de la evaluación no presentaba sintomatología ansiosa. En el BDI-II obtuvo una puntuación de 14 , lo que indica la presencia de sintomatología depresiva leve (Beck et al., 1996).

Con respecto a la evaluación de parámetros físicos antes del inicio de la intervención, F. M. pesaba $70 \mathrm{~kg}(\mathrm{IMC}=22)$, tenía una tensión arterial sistólica de 120 y Diastólica de 73 y una frecuencia cardíaca de 86 pulsaciones por minuto (ppm). Se evaluó también el nivel de CO, obteniendo 31 partículas por millón (p.p.m). 


\section{Intervención}

Se aplicó el programa psicológico cognitivo-conductual para dejar de fumar de Becoña (2007). Consta de seis sesiones con los siguientes componentes: contrato terapéutico, autorregistros, representación gráfica del consumo, información general sobre el tabaco, desvanecimiento (reducción gradual de la ingesta de nicotina y alquitrán), control de estímulos, estrategias para no padecer el síndrome de abstinencia de la nicotina, feedback fisiológico del consumo de cigarrillos (evaluación de monóxido de carbono en aire espirado) y entrenamiento en estrategias de prevención de la recaída (entrenamiento en asertividad, solución de problemas, cambio de creencias erróneas, manejo de la ansiedad y de la ira, ejercicio físico, control del peso y autorreforzamiento) y de mantenimiento de la abstinencia. Se trata de una intervención exclusivamente psicológica, en la que los participantes no reciben ayuda farmacológica de ningún tipo para dejar de fumar.

Este programa ha sido evaluado en distintos estudios, obteniendo porcentajes de abstinencia de entre el $58 \%$ y el $85 \%$ al final del tratamiento, y del 30\% al 54\% tras un año de seguimiento (Becoña \& Gómez-Durán, 1993; García \& Becoña, 2000). A continuación se describe sesión a sesión la evolución del presente caso:

\section{Primera sesión}

Al inicio de la sesión se entregó el material de las tareas a realizar durante la primera semana de intervención y la gráfica del consumo de cigarrillos que debía cubrir diariamente. Se evaluó el $\mathrm{CO}$ en aire espirado, mostrando una reducción con respecto a la evaluación, ya que obtuvo 15 p.p.m.

Para establecer la línea base de consumo, se usaron los autorregistros que había realizado en los días previos, siendo el número de cigarrillos que fumó el día anterior al inicio del tratamiento de 20 de $0,8 \mathrm{mg}$ de nicotina. Además, a través de los autorregistros, se realizó el análisis funcional de la conducta y se le explicó cuáles eran los antecedentes y consecuentes de su conducta de fumar y cómo reconocerlos.

Durante esta primera sesión se presentó información y datos objetivos sobre qué es el tabaco, sus componentes y consecuencias que produce en la salud, proporcionándole material escrito de apoyo. Asimismo, se discutieron las razones que le llevaban a fumar y cuáles eran los motivos por los que se había decidido en ese momento a dejar de fumar.

Se le explicó la técnica de desvanecimiento que se llevaría a cabo a lo largo de las siguientes semanas de intervención, y que consiste en el cambio de marca de cigarrillos, con el objetivo de reducir la ingestión de nicotina y alquitrán, y así lograr una disminución de la dependencia fisiológica. Se le indicó que a partir del día siguiente debía fumar una marca de cigarrillos que contuviera $0,6 \mathrm{mg}$ de nicotina y se estableció un máximo de 18 cigarrillos diarios. Para evitar la compensación nicotínica, que puede producirse debido a la reducción del consumo, se le indicó que debía evitar realizar inhalaciones profundas, que dejase un tercio del cigarriIlo sin fumar, y que no tuviera el cigarrillo en la boca mientras realizaba otras tareas.

Para lograr la adquisición de un compromiso social en el proceso de abandono, se le in- 
dicó que debía comunicar al menos a una persona de su entorno (familiar, amigo, etc.) que estaba llevando a cabo un intento de abandono del tabaco y que era posible que dejase de fumar en los próximos 30 días. Por último, se introdujo una norma orientada a la prevención de la recaída, y que consistía en que no podía aceptar ofrecimientos de cigarrillos. El objetivo principal de la misma era que aprendiese a rechazar dichos ofrecimientos ya que es una de las principales causas de la recaída.

\section{Segunda sesión}

Se revisaron los autorregistros realizados durante la semana previa, y se observó que F. M. había respetado el tope máximo, e incluso algún día de la semana había fumado menos del tope. El nivel de CO en esta segunda sesión fue de 15 p.p.m.

Se analizaron los problemas que habían surgido a lo largo de la semana y si había realizado adecuadamente las tareas que se habían señalado en la sesión anterior. Las situaciones en las que indicó tener más dificultades para controlar el consumo estaban asociadas o bien con momentos de estrés y ansiedad, o bien con situaciones asociadas con estados emocionales positivos, como estar con amigos que fuman.

Se realizó un nuevo cambio de marca, por lo que esa semana fumaría una marca que contuviese 0,4 mg de nicotina y se establecieron 15 cigarrillos al día como número máximo.

En esta sesión se introdujo la técnica del control de estímulos. Dado que los fumadores tienen condicionado su consumo a distintas situaciones, personas, pensamientos, estados emocionales, etc., dicha técnica tiene por objetivo debilitar la asociación entre la conducta de fumar y distintas situaciones y, con el tiempo, reducir la dependencia psicológica establecida. Después de explicar la racionalidad de la técnica, F. M. seleccionó tres situaciones en las que dejaría de fumar a partir del día siguiente. Concretamente eligió viendo la televisión, cocinando y caminando.

Con el objetivo de reducir los síntomas del síndrome de abstinencia de la nicotina que pudieran producirse a lo largo de la semana, se le explicaron diferentes estrategias para hacerles frente. Entre ellas se encontraban beber abundantemente tanto agua, como zumos naturales o infusiones, reducir el consumo de estimulantes y alcohol, realizar actividad física como caminar, tomar chicles o caramelos sin azúcar, etc.

Por último se le explicó la técnica de la respiración profunda, que trata de lograr una reducción de la activación del organismo. Se le pidió que la pusiese en práctica varias veces al día a lo largo de toda la semana, y así lograr el dominio en el manejo de la técnica. De esta forma, podría usar la respiración para manejar no sólo de las ganas de fumar, sino también otras situaciones estresantes del día a día, y que en su caso, eran un claro antecedente de la conducta de fumar.

\section{Tercera sesión}

Se revisaron las dificultades de la semana previa y si había realizado adecuadamente las tareas. Los autorregistros realizados durante la semana previa indicaron que había cumplido el tope máximo y que no había fumado 
en las situaciones que había escogido. De hecho, acudió a la sesión sin fumar, obteniendo un nivel de $\mathrm{CO}$ de tan solo 3 p.p.m. Él mismo propuso realizar un intento de mantenerse sin fumar durante la semana siguiente, ya que refirió tener dificultades respiratorias y molestias cuando fumaba.

En cuanto a las reacciones que produjo en las personas de su entorno el conocimiento de que iba a dejar de fumar y el apoyo que recibió de las mismas, F. M. indicó que su pareja lo apoyó y animó durante toda la semana, lo cual era realmente importante para él.

Durante la sesión se trató el tema de la ganancia de peso asociada a dejar de fumar. En este caso concreto, era importante que el peso se mantuviese sin cambios significativos, ya que el sobrepeso es un factor de riesgo para los problemas cardiovasculares. Para abordar este tema, se le indicaron una serie de estrategias básicas de alimentación para controlar el peso y varias estrategias conductuales complementarias como por ejemplo: intentar comer despacio, aprender a comer menos y controlar la conducta de sobreingesta. Además también se le explicaron los beneficios de realizar actividad física y se realizó una programación de la actividad física a realizar durante la siguiente semana.

\section{Cuarta sesión}

Acudió a esta sesión sin haber fumado a lo largo de toda la semana previa, y obtuvo 2 p.p.m. de CO. Refirió haber tenido ganas de fumar en ciertas ocasiones relacionadas especialmente con el estrés, la ansiedad y el estado de ánimo bajo, pero que era capaz de manejar- las mediante la aplicación de la estrategia de la respiración profunda.

En esta sesión se trabajó con el concepto de verse como un no fumador, y se realizó una proyección futura en imaginación de cómo sería mantenerse abstinente a largo plazo. Se explicó la distinción entre caída y recaída, y se introdujo el concepto de efecto de violación de la abstinencia, para una mejor comprensión del proceso de recaída. El objetivo de introducir estos conceptos es prevenir la recaída y conseguir así que, una vez abstinente, si tuviese algún desliz no le llevase a volver al nivel de consumo de cigarrillos inicial. Se realizó un entrenamiento para reconocer las tentaciones e impulsos a fumar que tuvo en la semana anterior y se analizaron las estrategias que había empleado para manejarlos, siendo la respiración profunda y el cambio de actividad las que le resultaron más útiles. También se le explicó que con el tiempo, dichos impulsos serían menos frecuentes, menos intensos y más breves.

Al final de la sesión se le entrenó en la técnica de solución de problemas (Tabla 1), con la finalidad de que mediante esta técnica mejorase la toma de decisiones ante situaciones problemáticas y así evitar que en el futuro se utilizase el tabaco como una estrategia de afrontamiento ante diferentes situaciones.

\section{Quinta sesión}

Acudió de nuevo sin haber fumado a lo largo de toda la semana previa, y obteniendo 2 p.p.m. de CO. Refirió tener cada vez menos ganas de fumar y una menor frecuencia de las mismas. Indicó que lo que más le ayudaba era beber agua y la aplicación de la respiración 
Tabla 1

Fases de la técnica de solución de problemas

\begin{tabular}{|c|c|c|}
\hline Fases & Descripción & Estrategias \\
\hline $\begin{array}{l}\text { 1. Orientación hacia el pro- } \\
\text { blema }\end{array}$ & $\begin{array}{l}\text { Reconocimiento de } \\
\text { la existencia de los } \\
\text { problemas }\end{array}$ & $\begin{array}{l}\text { Reconocer que los problemas son cotidianos y que } \\
\text { forman parte de nuestra vida normal }\end{array}$ \\
\hline 2. Definición del problema & $\begin{array}{l}\text { Especificar de } \\
\text { manera clara } \\
\text { y operativa las } \\
\text { características del } \\
\text { problema }\end{array}$ & $\begin{array}{l}\text { Formulación de preguntas como ¿quién tiene el } \\
\text { problema?, ¿por qué es un problema para mí?, ¿a qué } \\
\text { áreas de mi vida afecta?, ¿a qué otras personas afecta? }\end{array}$ \\
\hline 3. Búsqueda de soluciones & $\begin{array}{l}\text { Generar tantas } \\
\text { soluciones como sea } \\
\text { posible sin valorarlas }\end{array}$ & $\begin{array}{l}\text { Generar soluciones concretas, no vagas ni abstractas, y } \\
\text { que se puedan llevar a cabo }\end{array}$ \\
\hline $\begin{array}{l}\text { 4. Valoración de las alterna- } \\
\text { tivas de solución y toma de } \\
\text { decisiones }\end{array}$ & $\begin{array}{l}\text { Evaluar cada } \\
\text { alternativa generada, } \\
\text { para poder decidir } \\
\text { cuál es la más } \\
\text { adecuada en ese } \\
\text { momento }\end{array}$ & $\begin{array}{l}\text { Considerar los pros y los contras de cada una de las } \\
\text { alternativas, buscando las ventajas y desventajas que } \\
\text { implicarían a nivel personal. Una vez realizado este } \\
\text { proceso, se toma la decisión de cual poner en marcha en } \\
\text { función de las circunstancias }\end{array}$ \\
\hline 5. Aplicación y revisión & $\begin{array}{l}\text { Diseño del plan de } \\
\text { acción, aplicación de } \\
\text { la solución y revisión } \\
\text { de su utilidad }\end{array}$ & $\begin{array}{l}\text { Especificar qué se va a hacer, cómo y cuándo diseñando } \\
\text { un plan de acción. } \\
\text { Una vez puesta en marcha la solución, revisar si ha sido } \\
\text { útil para resolver el problema } \\
\text { Si el resultado no es el esperado, deberemos examinar } \\
\text { todo el proceso hasta encontrar el error y tratar de } \\
\text { corregirlo. En ocasiones existen factores ajenos a la } \\
\text { persona que pueden influir en la eficacia de la estrategia } \\
\text { seleccionada }\end{array}$ \\
\hline
\end{tabular}

profunda. Manifestó sentirse muy satisfecho por todos los logros alcanzados y por haber conseguido mantenerse sin fumar.

A lo largo de la sesión se trabajó con las creencias erróneas relacionadas con el proceso de abandono de los cigarrillos, y se analizaron de nuevo las tentaciones o impulsos de fumar. Por último, se habló de las mejorías físicas que había experimentado (mejor olfato y gusto, mejor respiración, desaparición de las sibilancias nocturnas, menos fatiga al realizar esfuerzos físicos, menos tos, mejor circulación en manos y pies, etc.) y psicológicas, destacando la mejora del estado de ánimo, la satisfacción personal y una cierta sorpresa por sentir que este procedimiento para dejar de fumar le había supuesto un menor esfuerzo que en otras ocasiones.

\section{Sexta sesión}

Acudió a la sesión sin haber fumado en la semana previa y obtuvo 2 p.p.m. de CO. Durante esta sesión se dedicó un tiempo a hablar acerca de las consecuencias positivas que le había tenido dejar de fumar y se realizó un balance acerca de las ventajas y desventajas no solo de estar sin fumar en este momento sino también a largo plazo. Tal y como se hizo en la sesión previa, se insistió en la diferencia entre caída y recaída, y se analizaron de nuevo las tentacio- 
nes o impulsos de fumar y aquellas estrategias que estaba aplicando para superarlas.

Al final de la sesión, se evaluó el peso, la tensión arterial y las pulsaciones por minuto en reposo, y además completó el Cuestionario de Evaluación Final de Tratamiento (Becoña, 1994) que incluye preguntas sobre el estatus de fumador, apoyo social, mejora física y psicológica, empeoramiento de algún aspecto, y el cuestionario BDI-II.

Con respecto a los parámetros físicos, se observó que no había ganado peso, que los valores de tensión arterial se mantuvieron estables y que se había producido una reducción en las pulsaciones por minuto en reposo (Tabla 2).

En el cuestionario, indicó sentirse muy satisfecho por haber conseguido dejar de fumar, para lo que había tenido un apoyo total de su entorno, y señaló una total confianza en mantenerse sin fumar. Además, su médico le reforzó el que hubiera logrado dejar de fumar, lo cual supuso una gran motivación para F. M.

Entre los beneficios que experimentó al dejar de fumar destacó la mejor capacidad respiratoria, la mejoría de la circulación, tener menos fatiga y una mejora en la percepción de sabores y olores. Además, destacó la importancia de las mejoras a nivel psicológico, ya que al haber logrado algo que era muy difícil, su estado de ánimo, su autoestima y autoeficacia se incrementaron significativamente. Respecto a la puntuación obtenida en el BDI-Il ésta se redujo, pasando de 14 en la evaluación inicial a 1 en esta última sesión de intervención.

\section{Seguimientos}

Durante dichos seguimientos F. M. indicó que se mantuvo sin fumar ni una sola calada desde la última sesión de intervención. Dicha abstinencia se validó bioquímicamente, tal y como puede observarse en la Figura 1.

Por otro lado, refirió que apenas había tenido tentaciones o impulsos de fumar y señaló que había experimentado muchas mejoras a nivel físico y psicológico (ej., menos fatiga y cansancio, mejor capacidad respiratoria, mejorías en el sueño y en la percepción de olores y sabores y una mejora también en la calidad de vida, en el estado de ánimo, en la autoestima y autoeficacia). Además, sus puntuaciones en sintomatología depresiva evaluada con el BDI-II, se mantuvieron bajas en todas las sesiones de seguimiento, pasando de 14 en la evaluación inicial a 1 al finalizar la misma y a 0 en los seguimientos realizados a lo largo de todo el año.

Tabla 2

Evolución del peso, tensión arterial y pulsaciones por minuto

\begin{tabular}{|c|c|c|c|c|c|}
\hline & $\begin{array}{c}\text { Evaluación pre } \\
\text { intervención }\end{array}$ & $\begin{array}{c}\text { Final de la } \\
\text { intervención }\end{array}$ & $\begin{array}{l}\text { Seguimiento } \\
3 \text { meses }\end{array}$ & $\begin{array}{c}\text { Seguimiento } \\
6 \text { meses }\end{array}$ & $\begin{array}{c}\text { Seguimiento } \\
12 \text { meses }\end{array}$ \\
\hline Peso en kg. (IMC) & $70(22)$ & $69(21,7)$ & $76(23,9)$ & $79,3(25)$ & $77,5(24,4)$ \\
\hline TAS & 120 & 110 & 127 & 124 & 115 \\
\hline TAD & 73 & 76 & 74 & 80 & 75 \\
\hline PPM & 86 & 71 & 72 & 77 & 67 \\
\hline
\end{tabular}

Nota: IMC = Índice de Masa Corporal; TAS = Tensión Arterial Sistólica; TAD = Tensión Arterial Diastólica; PPM = Pulsaciones Por Minuto 


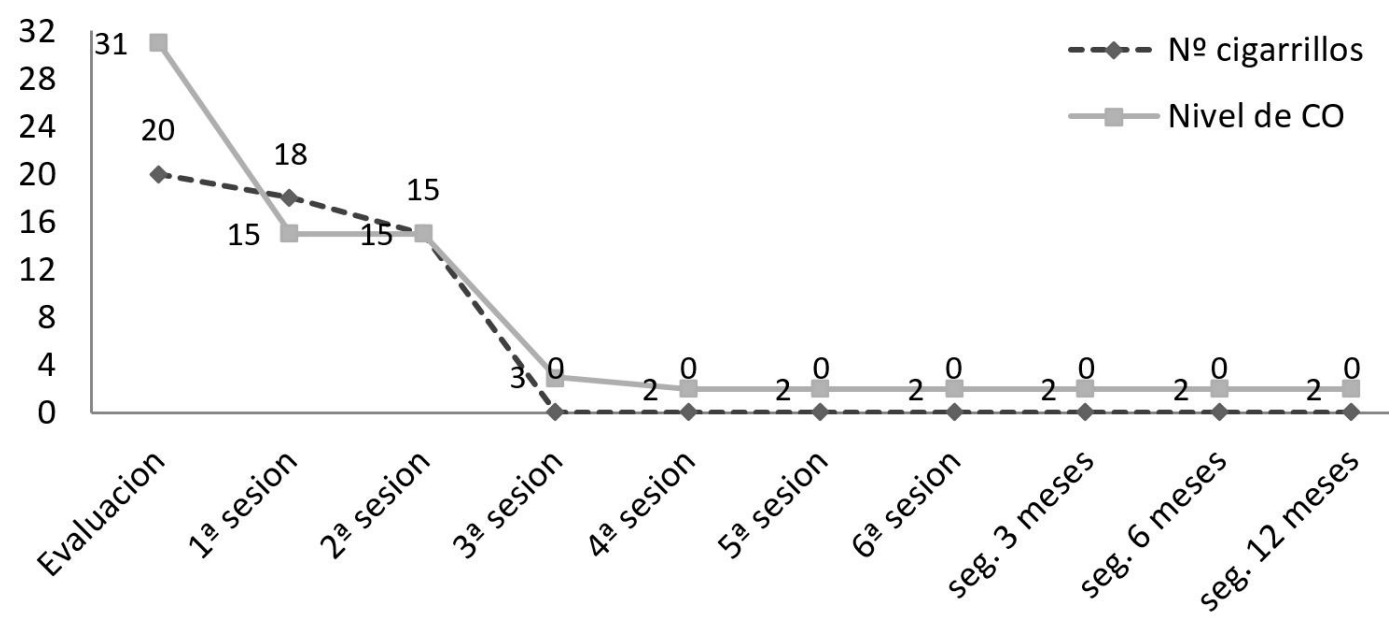

Figura 1. Evolución del consumo de tabaco (no de cigarrillos/día) y nivel de monóxido de carbono (CO) en aire espirado en partículas por millón en la evaluación, sesiones de intervención y seguimientos.

Con respecto a los parámetros físicos evaluados en los seguimientos realizados a los 3, 6 y 12 meses, se observó un ligero incremento del peso (aunque siempre manteniéndose dentro de los valores del IMC considerados normopeso, entre 20 y 25), un mantenimiento de los valores de tensión arterial y una disminución importante de las pulsaciones por minuto (ver Tabla 2). Este último dato resulta muy significativo debido a los problemas cardiovasculares que presentaba F. M., ya que las pulsaciones por minuto en reposo elevadas, especialmente en el caso de los varones, predicen no solo una mayor probabilidad de padecer una enfermedad cardiovascular, sino también un peor pronóstico si ya está presente, así como una mayor probabilidad de muerte prematura (Woodward et al., 2014).

\section{Discusión y conclusiones}

El objetivo del presente estudio de caso único es describir una intervención cognitivoconductual para dejar de fumar en una persona que padecía enfermedades relacionadas directamente con el consumo de tabaco y que ponían en riesgo su salud física, psicológica y su calidad de vida.

Un síndrome coronario agudo, como la angina de pecho, resulta un evento médico significativo que conduce a algunas personas a realizar cambios en el estilo de vida como por ejemplo dejar de fumar. Si además está presente una enfermedad respiratoria, el abandono del consumo de tabaco se convierte en algo más relevante si cabe. En la actualidad se siguen realizando estudios respecto a la seguridad del tratamiento farmacológico para dejar de fumar en personas con problemas cardiovasculares y respiratorios. Sin embargo, se sabe que las intervenciones psicológicas para dejar de fumar no solo son completamente seguras, sino que además su eficacia ha sido probada en múltiples estudios (Arias, García-Vera, \& Sanz, 2014).

El presente caso es una muestra de que las intervenciones cognitivo-conductuales son útiles para el logro de la abstinencia del tabaco y su mantenimiento a largo plazo, ya que se han presentado datos de seguimiento un año después de la realización de la intervención. Es 
más, la persona no solo logró dejar de fumar, sino que además también se produjo una mejoría importante en su estado emocional y calidad de vida. Aunque en este caso la sintomatología depresiva previa a la intervención para dejar de fumar era leve, se sabe que su presencia tras un evento coronario está relacionada con un peor pronóstico y un mayor riesgo de resultados adversos a largo plazo (Lichtman et al., 2014). En este caso, dejar de fumar resultaba de gran importancia, no solo porque el tabaco supone un factor de riesgo para las enfermedades físicas presentes sino también debido a la mejora del estado de ánimo que se produjo tras dejar de fumar y las implicaciones que esto tiene en pacientes con enfermedades cardiovasculares (Sin, Kumar, Gehi, \& Whooley, 2016).

A pesar de la relevancia de los datos presentados, al tratarse de un estudio de caso único no podemos obviar sus limitaciones. Es decir, la capacidad de generalizar dichos resultados a toda la población de fumadores con enfermedades físicas concurrentes es limitada. Por otro lado, sufrir un evento coronario agudo puede considerarse un momento ideal para dejar de fumar, debido a la elevada motivación del paciente (Tofler, May, Bartrop, Kirkness, Glinatsis, \& de Burgh, 2015). Este hecho podría generar dudas acerca de si el logro de la abstinencia es debido a dicho suceso, o a la intervención. Sin embargo, es importante destacar que si bien es cierto que la motivación influye en el proceso de abandono del tabaco, existen estudios que informan que por sí sola no es suficiente para lograr y mantener la abstinencia a largo plazo (Johnston, Johnston, Pollard, Kinmonth, \& Mant, 2004), y que más de la mitad de los pa- cientes fumadores con enfermedad coronaria continúan fumando después del diagnóstico de la misma e incluso después del alta hospitalaria tras haber padecido un evento coronario agudo (Berndt et al., 2012).

Se concluye que una adecuada intervención cognitivo conductual para dejar de fumar como la que se presenta resulta útil para dejar de fumar y mantener la abstinencia a largo plazo, logrando con ello una mejora en el pronóstico de problemas cardiovasculares y respiratorios, en la salud física general y psicológica y en la calidad de vida. Además los resultados obtenidos revelan la necesidad de seguir investigando la eficacia de las intervenciones cognitivo-conductuales, especialmente en aquellas poblaciones más vulnerables.

\section{Referencias}

Almirall, J., Blanquer, J., \& Bello, S. (2014). Neumonía adquirida en la comunidad en fumadores. Archivos de Bronconeumología, 50, 250-254. doi: 10.1016/j.arbres.2013.11.016

Arias, I. G. F., García-Vera, M. P., \& Sanz, J. (2014). Cuanta más psicología, mejor: eficacia para dejar de fumar de la terapia cognitiva conductual intensiva y de los parches de nicotina combinados con terapia cognitiva conductual intensiva y menos intensiva: Primer premio de la XX edición del Premio de Psicología Aplicada "Rafael Burgaleta" 2013. Clínica y Salud, 25, 1-10. doi: 10.5093/ cl2014a7

Beck, A., Steer, R. A., \& Brown, G. K. (1996). BDIII. Beck Depression Inventory-Second Edition. Manual. San Antonio, TX: The Psychological Corporation.

Becoña, E. (1994). Evaluación de la conducta de fumar. En J. L. Graña (Ed.), Conductas Adictivas: Teoría, evaluación y tratamiento (pp.403-454). Madrid: Debate.

Becoña, E. (2007). Programa para Dejar de Fumar. Vigo: Nova Galicia Edicións. 
Becoña, E., Fernández del Río, E., López-Durán, A., Míguez M. D. C., Castro, J., Nogueiras L. ... Vázquez, D. (2011). La escala breve de evaluación del síndrome de dependencia de la nicotina (NDSS-S) en fumadores españoles. Psicothema, 23, 126-132.

Becoña, E., \& Gómez-Durán, B. J. (1993). Programas de tratamiento en grupo de fumadores. En D. Macià, F. X. Méndez y J. Olivares (Eds.), Intervención psicológica: programas aplicados de tratamiento (pp. 203230). Madrid: Pirámide.

Becoña, E., \& Míguez, M. C. (1995). Abstinencia, recaída y no abandono en un programa para dejar de fumar, Adicciones, 7, 41-58.

Becoña, E., López-Durán, A., Fernández del Río, E., \& Martínez, Ú. (2014). Changes in the profiles of smokers seeking cessation treatment and in its effectiveness in Galicia (Spain) 2001-10. BMC Public Health, 14, 613. doi: 10.1186/1471-2458-14-613

Berndt, N., Bolman, C., Mudde, A., Verheugt, F., de Vries, H., \& Lechner, L. (2012). Risk groups and predictors of short-term abstinence from smoking in patients with coronary heart disease. Heart \& Lung: The Journal of Acute and Critical Care, 41, 332-343.

Doyle, F., Rohde, D., Rutkowska, A., Morgan, K., Cousins, G., \& McGee, H. (2014). Systematic review and meta-analysis of the impact of depression on subsequent smoking cessation in patients with coronary heart disease: 1990 to 2013. Psychosomatic Medicine, 76, 44-57.

García, M. P., \& Becoña, E. (2000). Evaluation of the amount of therapist contact in a smoking cessation program. The Spanish Journal of Psychology, 3, 28-36.

Hartmann-Boyce, J., Stead, L. F., Cahill, K., \& Lancaster, T. (2013). Efficacy of interventions to combat tobacco addiction: Cochrane update of 2012 reviews. Addiction, 108, 17111721. doi: 10.1111/add.12291

Instituto Nacional de Estadística (INE). (2018). Encuesta Nacional de Salud: ENSE 20162017. Recuperado a partir de https://www. mscbs.gob.es/estadEstudios/estadisticas/encuestaNacional/encuestaNac2017/ ENSE2017_notatecnica.pdf
Irvin, J. E., \& Brandon, T. H. (2000). The increasing recalcitrance of smokers in clinical trials. Nicotine \& Tobacco Research, 2, 79-84. doi: 10.1080/14622200050011330

Irvin, J. E., Hendricks, P. S., \& Brandon, T. H. (2003). The increasing recalcitrance of smokers in clinical trials II: Pharmacotherapy trials. Nicotine \& Tobacco Research, 5, 2735. Doi: 10.1080/1462220031000070534

Jha, P., Ramasundarahettige, C., Landsman, V., Rostron, B., Thun, M., Anderson, R. N., ... Peto, R. (2013). 21st-century hazards of smoking and benefits of cessation in the United States. New England Journal of Medicine, 368, 341-350. doi: 10.1056/NEJMsa1211128

Johnston, D. W., Johnston, M., Pollard, B., Kinmonth, A. L., \& Mant, D. (2004). Motivation is not enough: prediction of risk behavior following diagnosis of coronary heart disease from the theory of planned behavior. Health Psychology, 23, 533-538.

Leyro, T. M., Crew, E. E., Bryson, S. W., Lembke, A., Bailey, S. R., Prochaska, J. J., ... Hall, S. M. (2016). Retrospective analysis of changing characteristics of treatment-seeking smokers: implications for further reducing smoking prevalence. BMJ Open, 6(6), e010960.

Lichtman, J. H., Froelicher, E. S., Blumenthal, J. A., Carney, R. M., Doering, L. V., Frasure-Smith, N., ... \& Vaccarino, V. (2014). Depression as a risk factor for poor prognosis among patients with acute coronary syndrome: systematic review and recommendations: A scientific statement from the American Heart Association. Circulation, 129(12), 1350-1369.

McConnaughy, E. A., Prochaska, J. O., \& Velicer, W. F. (1983). Stages of change in psychotherapy: measurement and sample profiles. Psychotherapy: Theory, Research, and Practice, 20, 368-375. doi: 10.1037/h0090198

Messner, B., \& Bernhard, D. (2014). Smoking and cardiovascular disease significance: mechanisms of endothelial dysfunction and early atherogenesis. Arteriosclerosis, Thrombosis, and Vascular Biology, 34, 509-515.

OECD (2013). Health at a Glance, 2013: OECD Indicators. Recuperado de: http://dx.doi. org/10.1787/health_glance-2013-en 
Patnode, C. D., Henderson, J. T., Thompson, J. M., Senger, C. A., Fortmann, S. P., \& Whitlock, E. P. (2015). Behavioral counseling and pharmacological interventions for tobacco cessation in adults, including pregnant women: A review of reviews for the U. S. Preventive Services Task Force. Annals of Internal Medicine, 163, 608-621. doi: 10.7326/ M15-0171

Penninx, B. W. (2017). Depression and cardiovascular disease: epidemiological evidence on their linking mechanisms. Neuroscience \& Biobehavioral Reviews, 74, 277-286. doi: 10.1016/j.neubiorev.2016.07.003

Sanz, J., \& Vázquez, C., (2011). BDI-II: Inventario de Depresión de Beck-II: Manual. Madrid: Pearson.

Sin, N. L., Kumar, A. D., Gehi, A. K., \&Whooley, M. A. (2016). Direction of Association Between Depressive Symptoms and Lifestyle Behaviors in Patients with Coronary Heart Disease: the Heart and Soul Study. Annals of Behavioral Medicine, 50, 523-532. doi:10.1007/ s12160-016-9777-9

Siu, A. L. (2015). Behavioral and pharmacotherapy interventions for tobacco smoking cessation in adults, including pregnant women: US Preventive Services Task Force Recommendation Statement. Annals of Internal Medicine, 163, 622-634. doi: 10.7326/ M15-2023

Spielberger, C. D., Gorsuch, R. L., \& Lushene, R. (1982). Manual del Cuestionario de Ansiedad Estado-Rasgo (STAI). Madrid: TEA Ediciones.

Tofler, G. H., May, R., Bartrop, R., Kirkness, A., Glinatsis, H., \& de Burgh, S. (2015). Acute coronary syndrome as a teachable moment for smoking cessation. Journal of Smoking Cessation, 10, 5-11.

US Department of Health and Human Services. (2014). The health consequences of smoking - 50 years of progress: A report of the surgeon general. Atlanta, GA: US Department of Health and Human Services, Centers for Disease Control and Prevention, National Center for Chronic Disease Prevention and Health Promotion, Office on Smoking and Health, 17.

Velicer, W.F., DiClemente, C.C., Rossi, J.S., \& Prochaska, J.O. (1990). Relapse situations and self-efficacy: An integrative model. Addictive Behaviors, 15, 271-283. doi: 10.1016/03064603(90)90070-E

Velicer, W. F., Prochaska, J. O., Rossi, J. S., \& Snow, M. G. (1992). Assessing outcome in smoking cessation studies. Psychological Bulletin, 111, 23-41.

Watkins, L. L., Koch, G. G., Sherwood, A., Blumenthal, J. A., Davidson, J. R., O'Connor, C., \& Sketch, M. H. (2013). Association of anxiety and depression with all-cause mortality in individuals with coronary heart disease. Journal of the American Heart Association, 2(2), e000068.

West, R., Hajek, P., Stead, L., \& Stapleton, J. (2005). Outcome criteria in smoking cessation trials: Proposal for a common standard. Addiction, 100, 299-303. doi: 10.1111/j.13600443.2004.00995.x

Woodward, M., Webster, R., Murakami, Y., Barzi, F., Lam, T. H., Fang, X., ... Rodgers, A. (2014). The association between resting heart rate, cardiovascular disease and mortality: evidence from 112,680 men and women in 12 cohorts. European Journal of Preventive Cardiology, 21, 719-726. doi: $10.1177 / 2047487312452501$

Yusuf, S., Hawken, S., Ôunpuu, S., Dans, T., Avezum, A., Lanas, F.,... Lisheng, L. (2004). Effect of potentially modifiable risk factors associated with myocardial infarction in 52 countries (the INTERHEART study): Case-control study. Lancet, 364, 937-952. Doi: 10.006/ S0140-6736(04)17018-9 